\title{
Excessive sleepiness of Thai night shift workers in medical circumstances indexed by Epworth Sleepiness Scale
}

\author{
Phuttharaksa Phucharoen', Phakkharawat Sittiprapaporn² \\ ${ }^{1}$ Researcher, Brain Science and Engineering Innovation Research Group, School of Anti-Aging and Regenerative \\ Medicine, Mae Fah Luang University, Bangkok, Thailand, and Department of Anti-Aging Medicine, School of Anti-Aging \\ and Regenerative Medicine, Mae Fah Luang University, Bangkok, Thailand, ${ }^{2}$ Assistant Professor, Brain Science and \\ Engineering Innovation Research Group, School of Anti-Aging and Regenerative Medicine, Mae Fah Luang University, \\ Bangkok, Thailand, and Department of Anti-Aging Science, School of Anti-Aging and Regenerative Medicine, Mae Fah \\ Luang University, Bangkok, Thailand
}

Background: Excessive sleepiness is a cardinal symptom of many sleep disorders including shift work sleep disorder. As shift work sleep disorder is one type of the circadian rhythms sleep-wake disorders (CRSDs), it composes of symptoms of insomnia or excessive sleepiness associated with a recurring work schedule that intersections with the usual sleep timetable. Aims and Objective: The objective was to study the sleep propensity (SPs) in Thai medical staffs who are working in the hospital in Thailand. Materials and Methods: Ten participants included night shift workers with excessive sleepiness. Each participant was assessed by standardized measures of excessive sleepiness (Epworth sleepiness scale [ESS] $\geq 11$ ). Exclusions included clinical major medical problem, psychiatric, neurological problem, use of drugs other than alcohol, uncorrected serious vision issue, pregnancy and lactation, use of antibiotics and herbs during this study. The ESS item scores in this study are all assessments of different situational SPs. Results: The results showed that about $70 \%$ of the subjects answered slight chance of dozing, while $20 \%$ answered moderate chance of dozing for sitting and reading situation. Only $10 \%$ of the subjects would never doze. There were $60 \%$ of the subjects answered slight chance of dozing, while $10 \%$ answered moderate chance of dozing for as a passenger in a car for hour without a break of the ESS. There were $30 \%$ mentioned that they would never doze. Conclusion: The situations can be described in general terms but not completely, for they depend on the subject's perception of them. Although these preliminary findings represent a relatively information, it may not reflect all the SPs in Thai medical staffs who are working in hospital. It need further research to be done in the larger extended way.
http://nepjol.info/index.php/AJMS DOI: 10.3126/ajms.v10i5.24987 E-ISSN: 2091-0576 P-ISSN: 2467-9100

Key words: Sleep; Shift worker; Sleep Propensity

\section{INTRODUCTION}

In 1990, the International Labor Office Geneva (ILO) described working in shifts as a method of organization of working time in which workers succeed one another at the workplace so that the establishment can operate longer than the hours of work of individual workersat different daily and night hours. Shift work can work fulltime and part-time in countries worldwide. Shift work can work full-time and part-time in countries worldwide.
Three years later, the European Council Directive 93/104 (1993) announced that "concerning certain aspects of the organization of working time, shiftwork shall mean any method of organizing work in shifts whereby workers succeed each other at the same work stations according to a certain pattern. Shift worker shall mean any worker whose work schedule is part of shiftwork." In the scientific literature, shiftwork has been commonly contained any arrangement of regular working hours other than the standard daylight hours $(7 / 8 \mathrm{am}-5 / 6 \mathrm{pm})$. In 
industrialized countries it is approximate about 15-20\% of workers are engaged in shift work. ${ }^{1}$ Common working time schedules are morning, evening, night, and rotating shifts, respectively. However, rotating shifts usually include occasionally changing and irregular work hours that depend on employer needs. ${ }^{1}$

Following data from the National Health Interview Survey Occupational Health Survey in 2010, there were about 29\% of all U.S. workers working as shift worker. Obviously, food preparation and serving occupations had the highest prevalence of working a work shift which were almost $63 \%$. ${ }^{1,2}$ Previous studies reported that shift workers might have problem about their level of cognitive impairment. In addition, many shift workers had a misaligned circadian rhythm with follow to their night work timetable. ${ }^{2,3}$ As night shift work hours, which generally cover among 21:00 and 08:00, are associated with chronic shortening of sleep time $(<7 \mathrm{~h}$ per $24 \mathrm{~h}$ ), this caused the increased sleepiness and lose in its function. ${ }^{3}$ Moreover, excessive sleepiness during awakening hours was more usual in workers on the night shift rather than in those who normally work during the day. ${ }^{3}$ More importantly, changing in circadian timing which produced a misalignment between internal biological timing and the sleep-wake timetable showed more contribute to excessive sleepiness, impaired function, and disorganized sleep, respectively, in night-shift workers. ${ }^{3,4}$ Some additional reports also revealed that many shift workers had a misaligned circadian clock showing the decreased total sleep time in comparison with both evening workers and day workers. ${ }^{5,6}$ About $45 \%$ of night shift workers had excessive sleepiness affecting to their activities of daily life. ${ }^{3-6}$ However, not all people working in shift developed shift work sleep disorder, many factors including scheduling variances, shift frequency, shift timetable, family/social responsibilities, and alterations in sleep and circadian physiology could potentially disturb an individual's response to shift work and thus the progress of shift work sleep disorder. ${ }^{4}$ In addition, shift workers who had excessive sleepiness in their shift and/or clinically significant disorganized sleep associated with social, occupational, or the others, were at risk for shift work sleep disorder. ${ }^{3,4}$

Excessive sleepiness is a cardinal symptom of many sleep disorders including shift work sleep disorder (SWSD). SWSD is classified as a circadian rhythm sleep disorder. The circadian rhythms sleep-wake disorders (CRSDs) consequence when an individual's internal clock was impotent to keep appropriate alignment with the external factor, or social or work responsibilities force an individual to follow a schedule that isn't along with their internal clock. ${ }^{7}$ As shift work sleep disorder is one type of CRSDs, it composes of symptoms of insomnia or excessive sleepiness associated with a recurring work schedule that intersections with the usual sleep timetable. ${ }^{7}$

Adult patients who had sleep disorders associated with excessive daytime sleepiness including obstructive sleep apnea syndrome (OSAS), periodic limb movement disorder (PLMD), narcolepsy, idiopathic hypersomnia and other miscellaneous disorders, were assessed their sleep propensity by using the Epworth Sleepiness Scale (ESS). ESS uses a quite differentmethod for measuring sleep propensity based on the subject's retrospective reports of dozing behavior in eight situations that are commonly met in daily life. ${ }^{8}$ The severity of their chronic daytime sleepiness was the important aspect of each patient's assessment. ${ }^{8}$ ESS scores could distinguish patients with various sleep disorders from normal subjects which were correlated significantly with the severity of obstructive sleep apnea (OSA) because it has a high test-retest reliability and a high level of internal consistency among its eight items. ${ }^{8-11}$

This study then reported the relationship between sleep propensities (SPs) measured in different situations according to the Epworth Sleepiness Scales (ESS). This study was Intended to examine the question of how far the sleepiness that such tests measure can be generalized todaily life of night shift workers in medical circumstances in Thailand.

\section{MATERIALS AND METHODS}

\section{Participants}

The study was done with ten participants, aged between 25-45 years old, who were night shift workers with excessive sleepiness. Inclusion criteria included night shift workers working at least 5 night shifts per month (each shift $\leq 12$ hours, with $\geq 6$ hours of each shift worked between 24.00 and 05.00 ) with a minimum duration of shift work $\geq 3$ months. The meeting criteria of standard measures of excessive sleepiness assessed by Epworth sleepiness scale $[\mathrm{ESS}] \geq 10$ were assigned to participation. Exclusion criteria included (1) major medical problem such as renal failure, cirrhosis, heart failure, cerebrovascular diseases, (2) psychiatric or neurological problems, (3) history of head injuries or problems with hearing or vision, (4) history of alcohol/drug abuse, (5) any CNS active medications or any drugs that could cause sedation in 1 week prior to study participant, (6) pregnancy and lactation, (7) history of alcohol or drug abuse, (8) average total $24 \mathrm{hr}$. coffee consumption $>750 \mathrm{mg}$, (9) consumption of others dietary supplements and herbs during this study, (10) use of antibiotics during the course of this study, and (11) a history of side effects towards pro- or prebiotic supplements, respectively. After receiving information about the aims of the study, all subjects provided written consent to participate in the study that was performed according to 
the Helsinki declaration standards and was approved by the local institutional review board of Mae Fah Luang University, Thailand.

\section{Excessive sleepiness}

The excessive sleepiness was used in the present study. Excessive sleepiness was considered by difficulty maintaining wakefulness and/or an increased possibly to fall asleep when sleep was inappropriate. ${ }^{7}$ Some individuals used subjective expression such as drowsiness, languor, inertness, fatigue, sluggishness for explaining symptoms of excessive sleepiness. ${ }^{12}$ Sleepiness was measured using the Epworth Sleepiness Scale (ESS). The ESS has been shown to predict objectively measured excessive sleepiness in the general population. ${ }^{13} \mathrm{In}$ order to assess prevalence rates of excessive sleepiness, a sample-based cutoff score was also used. Previous studies have applied a score of 10 or higher on the ESS to indicate excessive sleepiness. ${ }^{14}$

\section{Epworth sleepiness scale (ESS)}

In this study, we determined the sleep propensity (SP) of Thai night shift workers who were working in the hospitals in Thailand in 2019. The ESS questionnaire and the method of its self-administration described in previous study ${ }^{8}$ were used in this study.The ESS was developed in 1991 by Dr. Murray Johns of the Epworth Hospital in Melbourne, Australia. ${ }^{15} \mathrm{It}$ is a self-administered questionnaire composed of eight questions with 4 levels of answers about the weekly frequency of that symptom. The lowest score was 0 point and the highest score was 24 points. It is commonly used to determine the level of daytime sleepiness. ${ }^{16} \mathrm{~A}$ score of greater than 10 is considered as sleepiness, and greater than 18 as excessive sleepiness. This score has been validated in prior studies ${ }^{17,18}$ and has been assessed to distinguish between clinical samples of individuals with and without sleep disorders. ${ }^{19}$ Eight questions or situations in the ESS which all subjects had to answer as followed:

Situation 1: Sitting and reading

Situation 2: Watching TV

Situation 3: Sitting, inactive in a public place (e.g., a theatre or a meeting)

Situation 4: As a passenger in a car for an hour without a break

Situation 5: Lying down to rest in the afternoon when circumstances permit

Situation 6: Sitting and talking to someone

Situation 7: Sitting quietly after a lunch without alcohol

Situation 8: In a car, while stopped for a few minutes in the traffic

The different situations in the questionnaire, referred to by their item numbers. In this study, we requested all subjects to answer by using the following scale to choose the most appropriate number for each situation:
$0=$ would never doze

$1=$ slight chance of dozing

$2=$ moderate chance of dozing

$3=$ high chance of dozing

\section{Statistical analysis}

Analyze the basic demographic data of participants by using descriptive analysis with qualitative data summarized in term of frequency and percentage, with quantitative data summarized in term of average and standard deviation. Descriptive analyses were performed for demographics utilizing characteristic measures such as percent, mean and standard deviation. Using inferential statistic compared each situation in the ESS by percentage.

\section{RESULTS}

With many factors and circumstances, only ten subjects participated in this study. According to the ESS, all subjects answers all eight situations one by one. About $70 \%$ of the subjects answered slight chance of dozing, while $20 \%$ answered moderate chance of dozing for sitting and reading situation. Only $10 \%$ of the subjects would never doze (see Figure 1).

Of the 10 subjects who answered situation 2 as Watching TV of Epworth Sleepiness Scale (ESS), about 60\% of the subjects would never doze while only $40 \%$ answered slight chance of dozing(see Figure 2).

In situation 3 as sitting, inactive in a public place of Epworth Sleepiness Scale (ESS), most of subjects would never doze while only $10 \%$ answered slight chance of dozing (see Figure 3).

On the other hand, there are $60 \%$ of the subjects answered slight chance of dozing, while $10 \%$ answered moderate

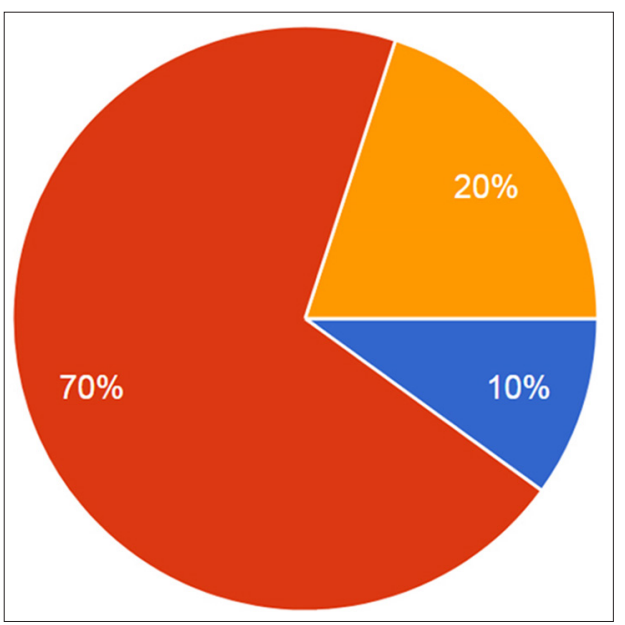

Figure 1: Situation 1: Sitting and reading of Epworth Sleepiness Scale (ESS) 
chance of dozing for as a passenger in a car for hour without a break of Epworth Sleepiness Scale (ESS). In the same way, there are $30 \%$ mentioned that they would never doze (see Figure 4).

For situation 5 as lying down to rest in the afternoon when circumstances permit, there are $60 \%$ the subjects answered slight chance of dozing, while $30 \%$ of the subjects mentioned that they would never doze. Moreover, there are $10 \%$ of the subjects answered moderate chance of dozing (see Figure 5).

It is surprisingly to learn that most of the subjects mentioned that they would never doze in sitting and talking to someone situation (see Figure 6).

However, about $60 \%$ of the subjects mentioned that they would never doze in sitting quietly after lunch without alcohol situation, but the data showed that there are $40 \%$ of the subjects answered slight chance of dozing (see Figure 7).

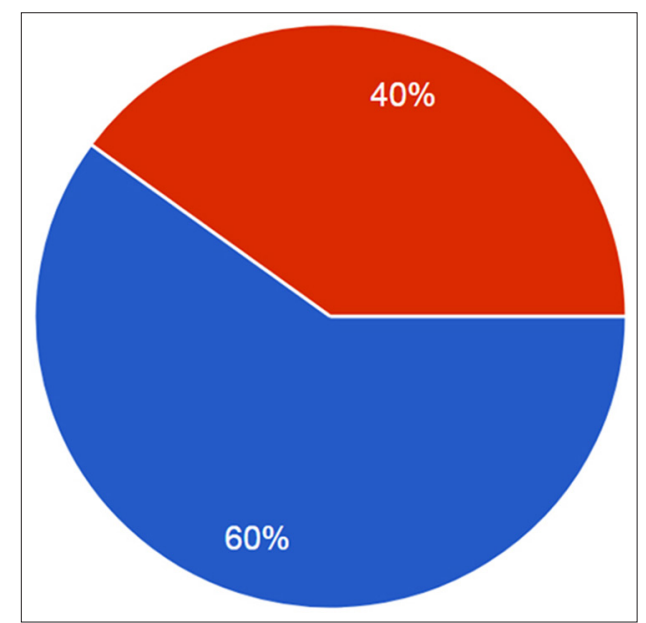

Figure 2: Situation 2: Watching tv of Epworth Sleepiness Scale (ESS)

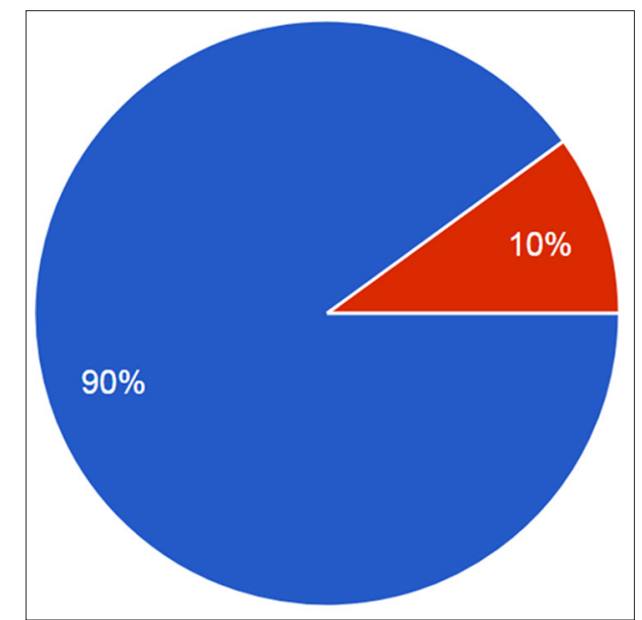

Figure 3: Situation 3: Sitting, inactive in a public place of Epworth Sleepiness Scale (ESS)
Finally, most of the subjects all mentioned that they would never doze in a car, while stopped for a few minutes in the traffic situation (see Figure 8).

\section{DISCUSSION}

This research has the purpose to study the sleep propensity (SP) in Thai night shift workers who were working in the hospital in Thailand. The results showed that about 70\% of the participants answered slight chance of dozing, while $20 \%$ answered moderate chance of dozing for sitting and reading situation. Only $10 \%$ of the participants would never doze (see Figure 1). On the other hand, there were $60 \%$ of the participants answered slight chance of dozing, while $10 \%$ answered moderate chance of dozing for as a passenger in a car for hour without a break of ESS. In the same way, there were $30 \%$ mentioned that they would never doze (see Figure 4). The results raise important issues about the concept and measurement of sleepiness.

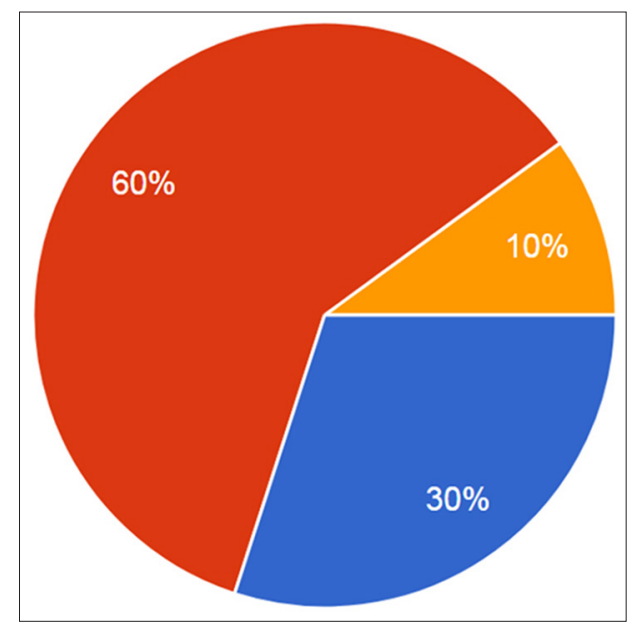

Figure 4: Situation 4: as a passenger in a car for hour without a break of Epworth Sleepiness Scale (ESS)

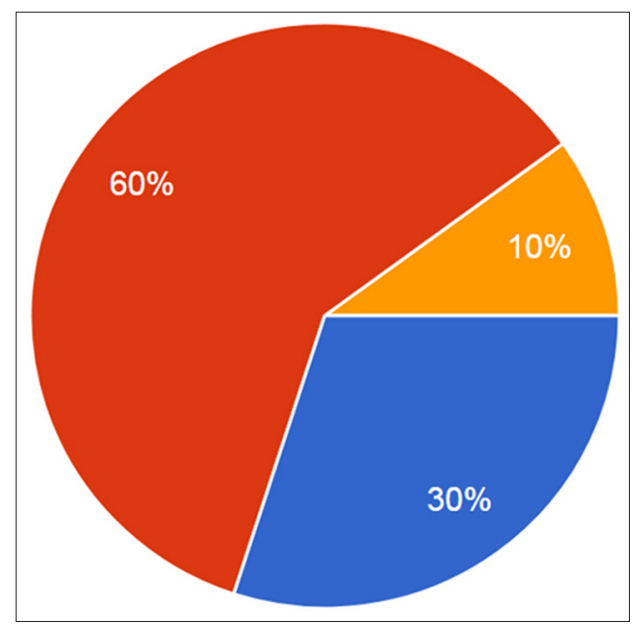

Figure 5: Situation 5: Lying down to rest in the afternoon when circumstances permit of Epworth Sleepiness Scale (ESS) 


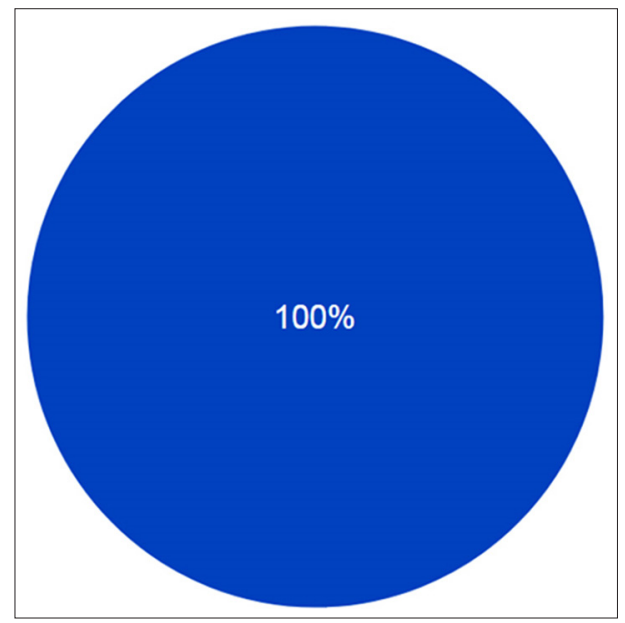

Figure 6: Situation 6: Sitting and talking to someone of Epworth Sleepiness Scale (ESS)

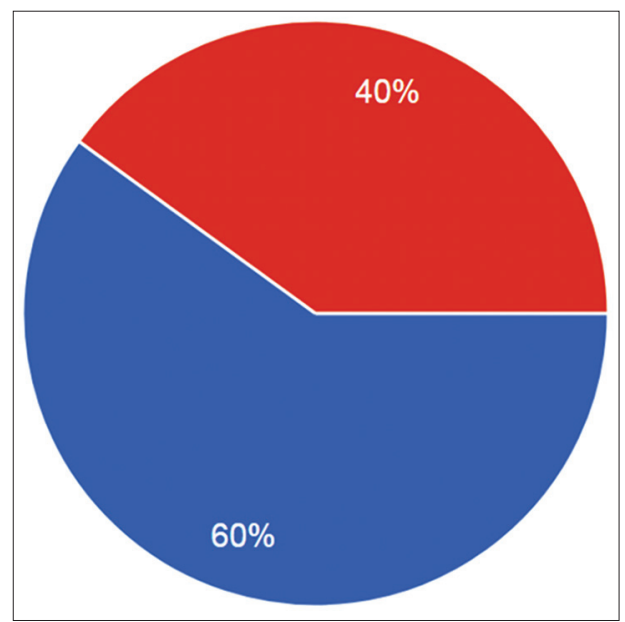

Figure 7: Situation 7: Sitting quietly after a lunch without alcohol of Epworth Sleepiness Scale (ESS)

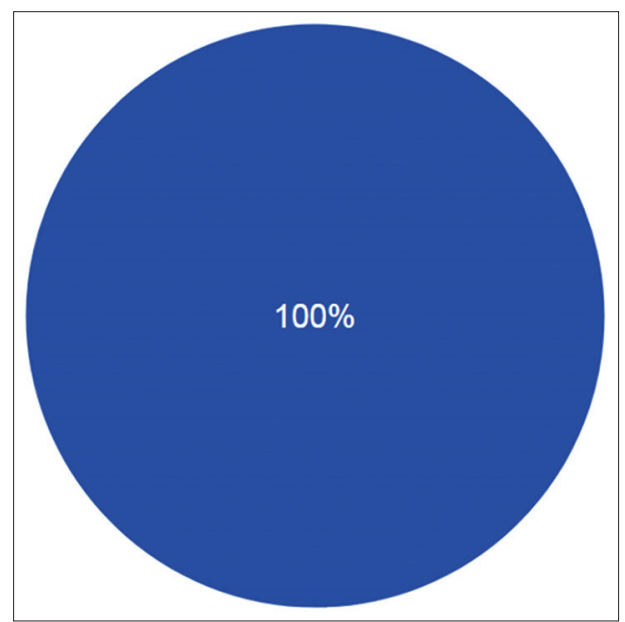

Figure 8: Situation 8: In a car, while stopped for a few minutes in the traffic of Epworth Sleepiness Scale (ESS)

The ESS item scores in this study were all assessments of different situational sleep propensity. The situations could be described in general terms but not completely, for they depended upon the participants' perception of them. It has been demonstrated previously how the sleep propensity in a particular situation could be influenced by instructions and motivation. ${ }^{19,20}$

The situational sleep propensity represented by the ESS item scoreswere relatively constant in a given participant and could be measured with a reliability. ${ }^{19,20}$ The total ESS scores provided an assessment of the subject's average sleep propensity that could be measured as reliably as the mean sleep latencyinthe multiple-sleep latency test (MSLT). ${ }^{21,22}$ It is of particular interest when assessing the effects of chronic sleep disorders such as obstructive sleep apnea (OSA) or narcolepsy on patients' sleepiness in daily life. ${ }^{21,22}$ For instant, many sleepy peoplesimply sit or lie downand relax almost anywhere caused them to fall asleep.The ESS could identify such people with a high sensitivity compared with the multiple-sleep latency test (MSLT). However, the ESS identified participants who claimed to be very sleepy in their daily lives but who did not fall asleep when under scrutiny in the multiple-sleep latency test (MSLT). Even though the nature of such a disorder remains unexplained, there is a substantial body of evidence that the multiple-sleep latency test (MSLT) revealed reliable, accurate and valid measurements of sleep propensity within its own test situation. ${ }^{21-23}$ Although this finding represent a relatively information, it may not reflect all the sleep propensity in Thai night shift workers who were working in hospital. It need further research to be done in the larger extended way.

Some previous studies revealed that excessive sleepiness was related with reduced cognitive and psychomotor function and decreased quality of life, as well as reduced physical and social functioning, emotional state, and mental health. Usually, individuals with excessive sleepiness have a reduced sense of well-being, often with fatigue and decreased energy levels. ${ }^{24-26}$ Moreover, some previous studies showed that there were important socioeconomic costs related with excessive sleepiness. ${ }^{4,24}$ Severe injury and death, costing billions of dollars every year, consequence from accidents caused by sleepiness. ${ }^{27}$ Consequently, excessive sleepiness was a factor in occupational accidents too, and patients with shift work sleep disorder were more probable to have accidents than daytime workers. ${ }^{4}$ Drake et $\mathrm{al}^{3}$ also found that individuals with shift work sleep disorder were at risk for significant behavioral and health-related morbidity related with their sleep-wake misalignment. Additional, it showed that the prevalence of shift work sleep disorder was about $10 \%$ of the night and rotating shift work population. ${ }^{4}$ 


\section{CONCLUSION}

The sleep propensity in Thai night shift workers who were working in the hospital in Thailand was assessed by standardized measures of excessive sleepiness (Epworth sleepiness scale $[E S S] \geq 11$ ). The situations could be described in general terms but not completely, for they depend upon the participants' perception of them. Although the present finding represents a relatively information, it might not reflect all the sleep propensity in Thai shift workers who were working in hospital. It need further research to be done in the larger extended way.

\section{ACKNOWLEDGEMENT}

We thank all of the participants who involved in this study as main data provider.

\section{REFERENCES}

1. Ker K, Edwards PJ, Felix LM, Blackhall K and Roberts I. Caffeine for the prevention of injuries and errors in shift workers. The Cochrane Database of Syst Rev 2010; (5): CD008508.

2. Alterman T, Luckhaupt SE, Dahlhamer JM, Ward BW and Calvert GM. Prevalence rates of work organization characteristics among workers in the U.S.: Data from the 2010 National Health Interview Survey. Am J Ind Med 2013; 56: 647-659.

3. Drake CL, Roehrs T, Richardson G, Walsh JK and Roth T. Shift work sleep disorder: prevalence and consequences beyond that of symptomatic day workers. Sleep 2004; 27:1453-1462.

4. Costa G. Factors influencing health and tolerance to shift work. Theor Issues Ergon Sci 2003; 263-288.

5. Pilcher JJ, Lambert BJ and Huffcutt Al. Differential effects of permanent and rotating shifts on self-report sleep length: Ametaanalytic review. Sleep 2000; 23:155-163.

6. Park YM, Matsumoto PK, Seo YJ, Cho YR and Noh TJ. Sleepwake behavior of shift workers using wrist actigraph. Psychiatry ClinNeurosci2000; 54(3):359-360.

7. American Academy of Sleep Medicine.The international classification of sleep disorders: diagnostic and coding manual. American Academy of Sleep Medicine, Westchester, III, 2005; 18: 297.

8. Johns MW. A new method for measuring daytime sleepiness: the Epworth Sleepiness Scale. Sleep1991; 14:540-545.

9. Smolley LB, Ivey C, Farkas M, Faucette E and Murphy S. Epworth Sleepiness Scale is useful in monitoring daytime sleepiness. Sleep Res.1993; 22: 389.

10. Roehrs T, Zorick F, Wittig R, Conway W and Roth T. Predictors of objective level of daytime sleepiness in patients with sleeprelated breathing disorders. Chest 1989; 95:1202-1206.

11. Johns MW. Reliability and factor analysis of the Epworth Sleepiness Scale. Sleep 1992; 15: 376-381.

12. Buysse DJ. Drugs affecting sleep, sleepiness, and performance. In: Monk TH, ed. Sleep, Sleepiness, and Performance. Chichester Wiley. 1991, 4-31.

13. Punjabi NM, Bandeen RK and Young T.Predictors ofobjective sleep tendency in the general population. Sleep 2003; 26:678-683.

14. Walsleben JA, Kapur VK, Newman AB, Shahar E, Bootzin RR, Rosenberg CE, et al. Sleep and reported daytime sleepiness in normal subjects: The Sleep Heart Health Study. Sleep 2004; 27(2):293-298

15. Alho K. Cerebral generators of Mismatch Negativity (MMN) and its magnetic counterpart (MMNm) elicited by sound changes. Ear Hearing 1995; 16:38-51.

16. Johns MW. A new method for measuring daytime sleepiness: the Epworth sleepiness scale. Sleep 1991; 14:540-545.

17. Chervin RD, Aldrich MS, Pickett $R$ and Guilleminault $C$. Comparison of the results of the Epworth Sleepiness Scale and the Multiple Sleep Latency Test. J Psychosom Res 1997; 42:145-155.

18. Johns MW. Reliability and factor analysis of the Epworth Sleepiness Scale. Sleep 1992; 15:376-381.

19. Johns MW.Sensitivity and specificity of the Multiple Sleep Latency Test (MSLT), the Maintenance of Wakefulness Test and the Epworth Sleepiness Scale: failure of the MSLT as a gold standard. J Sleep Res 2000; 9:5-11.

20. Alexander $C$, Blagrove $M$ and Home J. Subject motivation and the Multiple Sleep Latency Test (MSLT). Sleep Res 1991; 20: 403.

21. Doorenbos $A-Z$, Roehrs $T$, Schaeffer $M$ and Roth $T$. Testretest reliability of the MSL T. Sleep. 1988; 2: 562-565.

22. Seidel WF and Dement WC. The Multiple Sleep Latency Test: testretest reliability. Sleep Res. 1981; 10: 284.

23. Thorpy MT. The clinical use of the Multiple Sleep Latency Test. Sleep 1992; 15:268-276.

24. Reimer MA and Flemons WW.Quality of life in sleep disorders. Sleep Med Rev 2003; 7:335-349.

25. Pigeon WR, Sateia MJ and Ferguson RJ.Distinguishing between excessive daytime sleepiness and fatigue: toward improved detection and treatment. J Psychosom Res 2003; 54:61-69.

26. Thorpy MJ.Which clinical conditions are responsible for impaired alertness.Sleep Med 2005; 6:13-20.

27. Leger D.The cost of sleep-related accidents: a report for the National Commission on Sleep Disorders Research. Sleep 1994; 17:84-93.

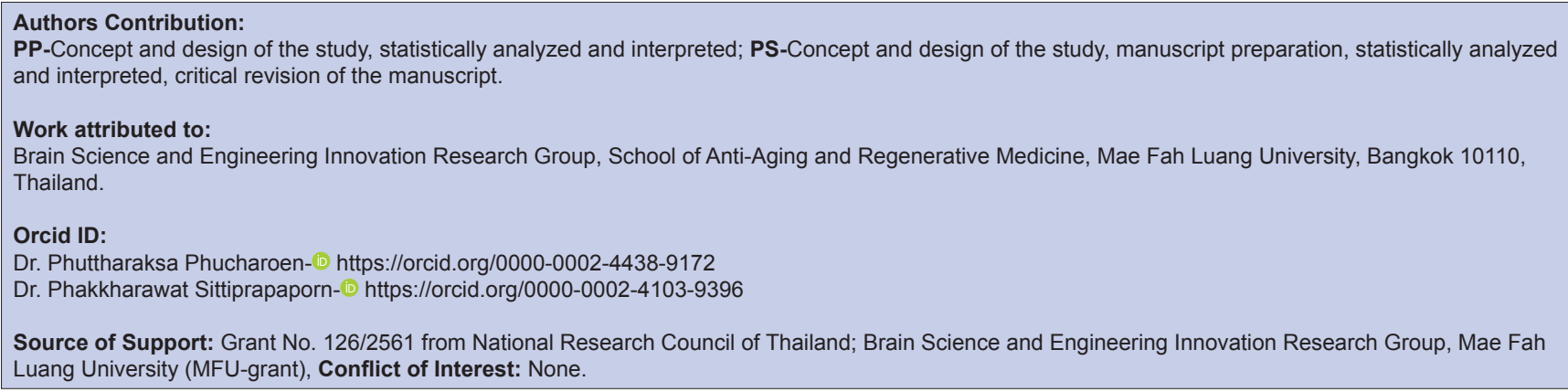

